\title{
EQUILÍBRIO LÍQUIDO-LÍQUIDO APLICADO A SEPARAÇÃO DE PRODUTOS DA PIRÓLISE DE BIOMASSA
}

\author{
A. C. C MELO, M. A. TEOTÔNIO, L. DANIELSKI, L. STRAGEVITCH \\ Universidade Federal de Pernambuco, Departamento de Engenharia Química \\ E-mail para contato: augusto.ccm@hotmail.com
}

\begin{abstract}
RESUMO - O bio-óleo corresponde à fração líquida obtida pela pirólise da biomassa. Além do seu uso como biocombustível, o bio-óleo possui em sua composição diversos compostos de interesse comercial. O processo de pirólise é um tratamento térmico em que a matéria orgânica é submetida ao aquecimento em meio normalmente inerte, na ausência ou em baixas concentrações de oxigênio. Com o objetivo de estudar a recuperação de compostos de valor comercial, neste trabalho foi medido o equilíbrio líquido-líquido (ELL) para o sistema quaternário água + ácido acético + ciclo-hexano + 1-butanol na temperatura de $303 \mathrm{~K}$. Os dados experimentais foram correlacionados utilizando o modelo UNIQUAC.
\end{abstract}

\section{INTRODUÇÃO}

A pirólise é um processo de decomposição térmica de materiais orgânicos na ausência de agentes oxidantes que fornece como produtos de reação uma fração líquida (bio-óleo), produtos gasosos e carvão vegetal. O processo que mais favorece esse produto é a pirólise rápida, a qual ocorre a altas temperaturas, porém, apresenta menor tempo de reação (BRIDGEWATER \& PEACOCKE, 2000). A composição e o rendimento do biocombustível são influenciados pelo tipo de material e pela tecnologia usada no processo de pirólise. O bio-óleo é formado basicamente por 20-30\% de água e 70-80\% de orgânicos polares (ALMEIDA, 2008).

A formação do bio-óleo se dá através da rápida e simultânea despolimerização da celulose, hemicelulose e lignina com o aumento da temperatura durante a pirólise (BRIDGEWATER, 2012). Este processo gera uma composição química complexa para este biocombustível, formada por aldeídos, ácidos, cetonas, álcoois e compostos fenólicos. A distribuição da massa molar desses componentes está relacionada com a taxa de aquecimento, tempo de residência, da espécie e tamanho das partículas, temperatura e biomassa utilizada (MOHAN et al., 2006).

O bio-óleo apresenta características físicas e energéticas semelhantes ao óleo diesel, podendo ser usado como combustível na queima direta em fornos, em substituição aos de diesel após passagem pelo processo de hidrogenação ou pode ser usado na produção de fertilizantes orgânicos e aditivos para combustíveis (GOMEZ, 2002). A energia produzida por este biocombustível é mais limpa que a gerada pelos combustíveis fósseis, pois, durante sua 
combustão, ele libera $\mathrm{CO}_{2}, \mathrm{SO}_{\mathrm{X}}, \mathrm{NO}_{\mathrm{X}}$ e materiais particulados em menor quantidade (SANTOS, 2013).

Existem vários compostos presentes no bio-óleo que apresentam valor comercial, como por exemplo, o ácido acético. Neste trabalho foram medidos dados de equilíbrio líquido-líquido (ELL) para o sistema quaternário água (1) + ácido acético (2) + ciclo-hexano (3) + 1-butanol (4) na temperatura de $303 \mathrm{~K}$. Esses dados são importantes para o projeto e simulação de uma unidade recuperação do ácido acético e de outros compostos do bio-óleo. Os dados medidos foram correlacionados através do modelo UNIQUAC.

\section{EXPERIMENTAL}

\subsection{Material}

Os reagentes utilizados foram: ácido acético (Sigma-Aldrich, $\geq 99,8 \%$ ), ciclo-hexano (Vetec, $\geq 99 \%$ ), 1-butanol (Panreac, $\geq 99,5 \%$ ), etanol (Carlo Erba, 99,9\%) e 1-pentanol (Merck, $\geq 99,5 \%$ ). A água destilada e deionizada utilizada em todos os experimentos foi preparada no laboratório.

\subsection{Procedimento}

O sistema foi mantido em uma célula de vidro encamisada, com a temperatura mantida por um banho termostático e medida por um termopar PT-100 com incerteza $\pm 0,1 \mathrm{~K}$. A mistura foi vigorosamente agitada por um agitador magnético por duas horas e deixada em repouso por mais duas horas para que houvesse a separação das fases e o equilíbrio fosse atingido. As amostras de ambas as fases foram coletadas com uma seringa de vidro.

A composição da amostra foi determinada por um cromatógrafo Shimadzu 17A com detector de ionização de chama e coluna capilar DB-WAX $(30 \mathrm{~m} \times 0,25 \mathrm{~mm} \times 0,25 \mu \mathrm{m})$ de fase estacionária $100 \%$ polietileno glicol. As temperaturas do injetor, da coluna e do detector foram 373, 338 e 463K, respectivamente. 1-pentanol foi utilizado como padrão interno e etanol como solvente para que não houvesse a separação de fases na amostra. A água foi determinada com um titulador coulométrico KF 831 da Metrohm.

\subsection{Modelagem}

O modelo Universal Quasi-Chemical - UNIQUAC (ABRAMS and PRAUSNITZ, 1975), foi usado para correlacionar os dados experimentais. As Equações 1-7 representam o modelo UNIQUAC e são dadas por:

$$
\begin{aligned}
& \ln \gamma_{i}=\ln \gamma_{i}^{\mathrm{C}}+\ln \gamma_{i}^{\mathrm{R}} \\
& \ln \gamma_{i}^{\mathrm{C}}=\ln \frac{\phi_{i}}{x_{i}}+\frac{z}{2} q_{i} \ln \frac{\theta_{i}}{\phi_{i}}+l_{i}-\frac{\phi_{i}}{x_{i}} \sum_{j} x_{j} l_{j}
\end{aligned}
$$




$$
\begin{aligned}
& \ln \gamma_{i}^{\mathrm{R}}=q_{i}\left[1-\ln \left(\sum_{j} \theta_{j} \tau_{j i}\right)-\sum_{j} \frac{\theta_{j} \tau_{i j}}{\sum_{k} \theta_{k} \tau_{k j}}\right] \\
& \phi_{i} \equiv \frac{x_{i} r_{i}}{\sum_{i}^{N} x_{i} r_{i}} \\
& \theta_{i} \equiv \frac{x_{i} q}{\sum_{i}^{N} x_{i} q_{i}} \\
& l_{i}=\frac{z}{2}\left(r_{i}-q_{i}\right)-\left(r_{i}-1\right) \\
& \tau_{j i}=\exp \left[-\left(\frac{u_{j i}-u_{i i}}{R T}\right)\right]
\end{aligned}
$$

\section{RESULTADOS E DISCUSSÃO}

\subsection{Composição e Linhas de Amarração}

\begin{tabular}{|c|c|c|c|c|c|c|c|}
\hline \multicolumn{4}{|c|}{ Fase Aquosa } & \multicolumn{4}{|c|}{ Fase Orgânica } \\
\hline $\mathrm{w}_{1}$ & $\mathrm{w}_{2}$ & $w_{3}$ & $\mathrm{w}_{4}$ & $\mathrm{w}_{1}$ & $\mathrm{w}_{2}$ & $\mathrm{w}_{3}$ & $\mathrm{w}_{4}$ \\
\hline 0,719 & 0,209 & 0,003 & 0,070 & 0,267 & 0,029 & 0,656 & 0,049 \\
\hline 0,579 & 0,330 & 0,007 & 0,084 & 0,105 & 0,034 & 0,842 & 0,019 \\
\hline 0,406 & 0,490 & 0,026 & 0,078 & 0,072 & 0,064 & 0,858 & 0,006 \\
\hline
\end{tabular}

A composição das fases em equilíbrio foi determinada para o sistema água + ácido acético + ciclo-hexano + 1-butanol a $303 \mathrm{~K}$ e a pressão atmosférica. A Tabela 1 apresenta as concentrações em fração mássica. Todos os dados foram obtidos em triplicata.

Tabela 1 - Dados experimentais do ELL para o sistema Água (1) +

A Figura 1 mostra o resultado da modelagem termodinâmica em comparação com os dados experimentais. Para uma melhor visualização gráfica as composições do ciclo-hexano e do 1butanol foram somadas e atribuídas como um só componente, caracterizando um sistema pseudoternário. Como pode ser observado, o sistema é do tipo I (TREYBAL, 1963), pois, água + (ciclo-hexano+1-butanol) são parcialmente miscíveis. 


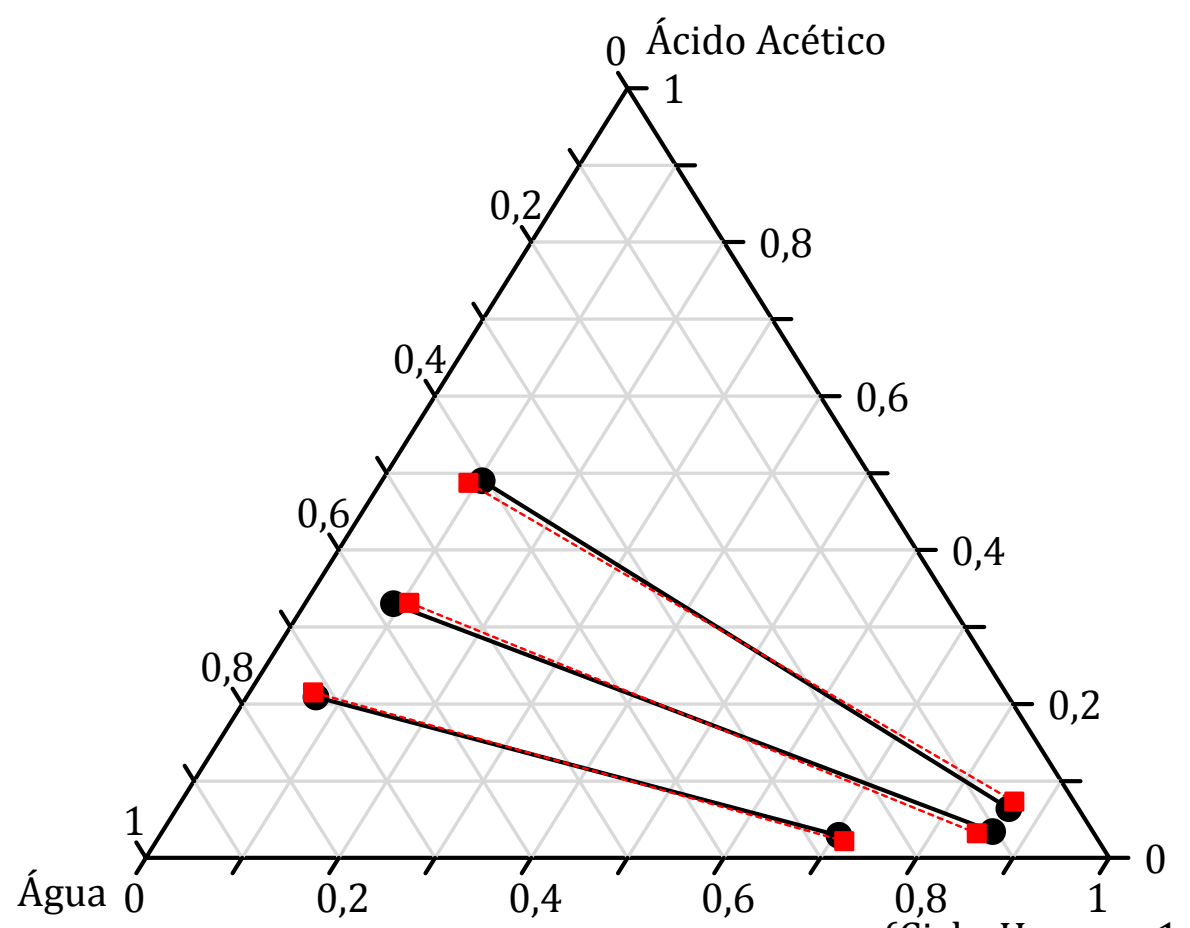

(Ciclo-Hexano+1-Butanol)

Figura 1 - ELL para o sistema água + ácido acético + (ciclo-hexano+1-butanol) a 303K.

$(\bullet$ dados experimentais; $\bullet$ UNIQUAC)

Observa-se uma boa separação entre o ácido acético e o 1-butanol, apesar da miscibilidade entre ambos. Com isso, a obtenção destes produtos mais puros é possível.

\subsection{Correlação}

Os dados experimentais foram correlacionados segundo a metodologia apresentada por STRAGEVITCH e D'ÁVILA (1997). O método SIMPLEX foi utilizado para regredir os parâmetros do modelo a partir dos dados empíricos. A Tabela 2 mostra os parâmetros estruturais UNIQUAC (PRAUSNITZ et al., 2001) e os parâmetros de interação binária estimados são mostrados na Tabela 3.

Tabela 2 - Parâmetros estruturais UNIQUAC

\begin{tabular}{ccc}
\hline Componente & $\mathrm{r}$ & $\mathrm{q}$ \\
\hline Água & 0,92 & 1,4 \\
Ácido Acético & 2,2024 & 2,072 \\
Ciclo-Hexano & 4,0464 & 3,24 \\
1-Butanol & 3,9243 & 3,668 \\
\hline
\end{tabular}


Tabela 3 - Parâmetros de interação

binária UNIQUAC

\begin{tabular}{cccc}
\hline $\mathrm{i}$ & $\mathrm{j}$ & $\mathrm{A}_{\mathrm{ij}}$ & $\mathrm{A}_{\mathrm{ji}}$ \\
\hline 1 & 2 & $-279,39$ & $-305,88$ \\
1 & 3 & 3000,0 & $-145,41$ \\
1 & 4 & $-16,142$ & 848,85 \\
2 & 3 & 422,76 & 5,5125 \\
2 & 4 & $-11,493$ & 229,61 \\
3 & 4 & 694,44 & $-97,289$ \\
\hline
\end{tabular}

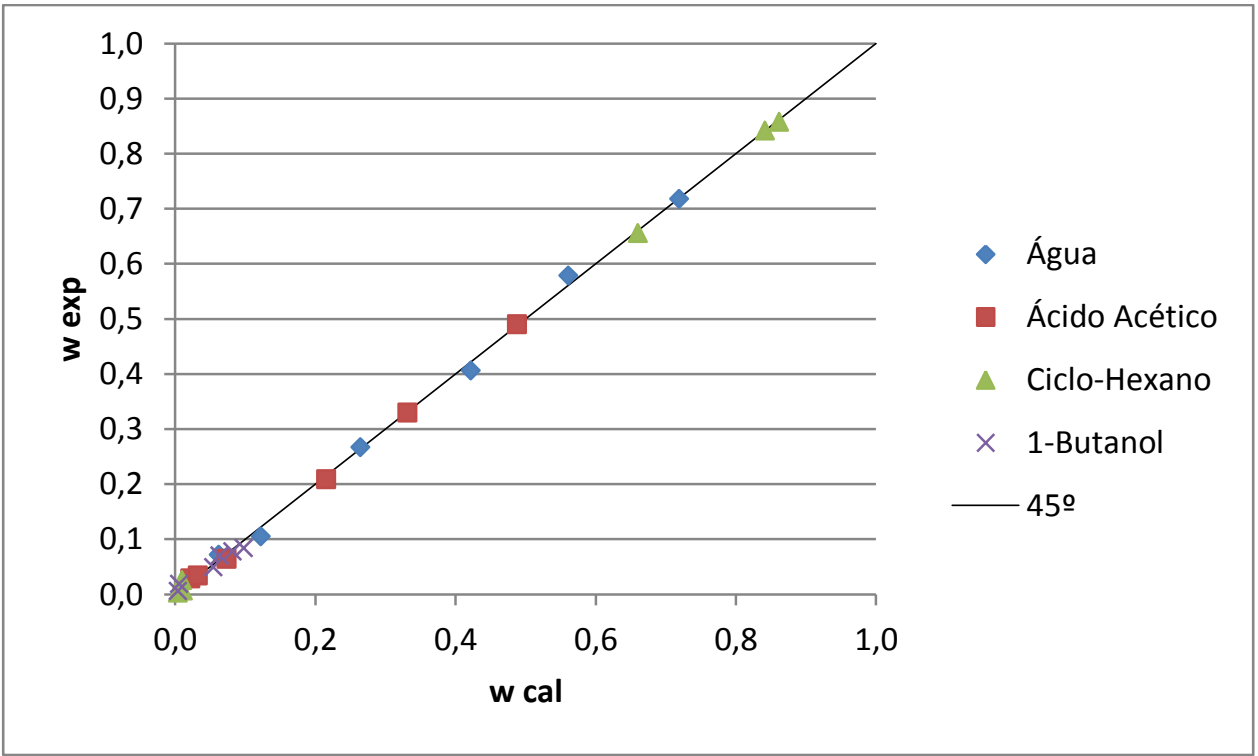

Figura 2 - Frações mássicas experimentais comparadas com os respectivos valores calculados

A Figura 2 ilustra o gráfico exp vs. cal para os componentes do sistema onde pode ser observada uma boa reprodutibilidade dos dados calculados. A Figura 3 mostra a dispersão dos desvios locais obtidos, e exceto o primeiro ponto para o 1-butanol, todos apresentaram desvios próximos ao eixo central. 


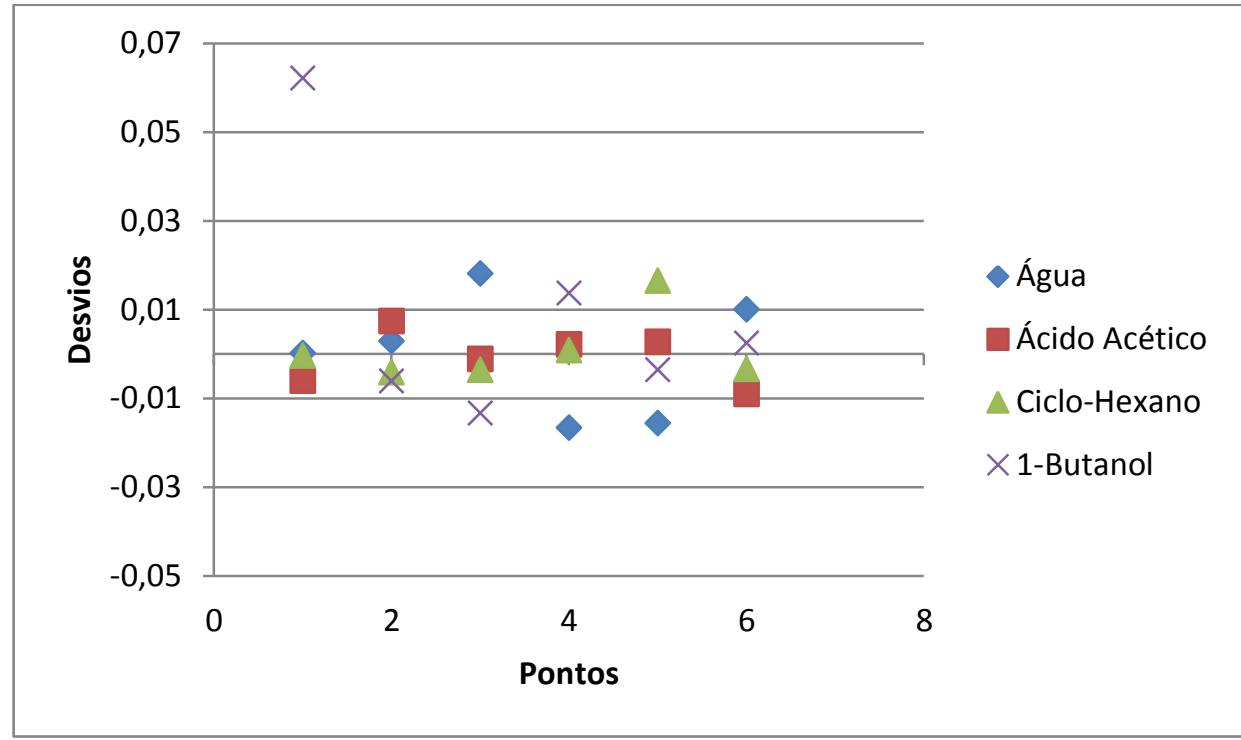

Figura 3 - Dispersão dos desvios das frações mássicas calculadas

\section{CONCLUSÃO}

Os dados do ELL para o sistema quaternário água + ácido acético + ciclo-hexano + 1butanol foram determinados a $303 \mathrm{~K}$. O sistema apresentou um diagrama de fases do tipo I. Os dados medidos foram satisfatoriamente correlacionados com o modelo UNIQUAC com um desvio quadrático médio igual a $0,89 \%$.

\section{NOMENCLATURA}

$\begin{array}{ll}\text { A } & \text { UNIQUAC parâmetros de interação binária } \\ \mathrm{N} & \text { Componente } \\ \mathrm{T} & \text { Temperatura }(\mathrm{K}) \\ \mathrm{R} & \text { Constante dos gases ideais } \\ \text { cal } & \text { Calculado } \\ \text { exp. } & \text { Experimental } \\ \mathrm{i}, \mathrm{j}, \mathrm{k} & \text { Componentes } \\ \mathrm{l} & \text { Ver Eq. (6) } \\ \mathrm{q} & \text { Parâmetro estrutural de área de van der Waals } \\ \mathrm{r} & \text { Parâmetros estrutural de volume de van der Waals } \\ \mathrm{u} & \text { interação binária } \\ \mathrm{w} & \text { fração mássica } \\ \mathrm{X} & \text { fração molar } \\ \mathrm{z} & \text { número de coordenação, definido como igual a } 10 \\ \gamma & \text { Coeficiente de atividade } \\ \theta & \text { Fração de área }\end{array}$


$\tau \quad$ Parâmetro de energia de interação binária

$\phi \quad$ Fração de volume

\section{REFERÊNCIAS}

ABRAMS, D. S., PRAUSNITZ, J. M., Statistical thermodynamics of liquid mixtures: A new expression for the excess Gibbs energy of partly or completely miscible systems. J. AIChE. vol. 21, p. 116-128, 1975.

ALMEIDA, M. B. B. Bio-óleo a partir da pirólise rápida, térmica ou catalítica da cana-deaçúcar e seu co-processamento com gasóleo em craqueamento catalítico. Dissertação de Mestrado: Escola de Química, UFRJ, 2008.

BRIDGWATER, A. V. Review of fast pyrolysis of biomass and product upgrading. Biomass and Bioenergy. vol. 38, p. 68-94, 2012.

BRIDGWATER, A. V., PEACOCKE, G. V. C. Fast pyrolysis processes for biomass. Renewable and Sustainable Energy Reviews. vol. 4, p. 1-73, 2000.

GÓMEZ, E.O. Estudo da pirólise rápida de capim elefante em leito fluidizado borbulhante mediante caracterização dos finos de carvão. Universidade Estadual de Campinas, Campinas, Tese de Doutorado, 2002.

PRAUSNITZ, J. M., POLING, B. E., O’CONNELL, J. P. The properties of gases and liquids. 5. ed. McGraw-HILL, 2001.

SANTOS, L. O. Estudo para obtenção de bio-óleo de $2^{\text {a }}$ geração através da pirólise de plantas aquáticas. Universidade Federal de Sergipe, Aracaju, Dissertação de Mestrado, 2013.

STRAGEVITCH, L.; D’ÁVILA, S.G. Application of a generalized maximum likelihood method in the reduction of multicomponent liquid-liquid equilibrium data. Braz. J. Chem. Eng., v. 14, p. 41-52. 1997.

TREYBAL, R. E., Liquid Extraction, second ed., McGraw-Hill, New York, 1963. 